\title{
Doenças respiratórias em crianças e adolescentes: um perfil dos atendimentos na atenção primária em Vitória/ES
}

\author{
Respiratory diseases in children and adolescents: profiling primary care visits in Vitória/ES \\ Enfermedades respiratorias en niños y adolescentes: un perfil de asistencia en la atención \\ primaria en Vitória/ES
}
Diana de Oliveira Frauches ${ }^{a}$, Isabela Bittencourt Coutinho Lopesa ${ }^{a}$, Henrique Ton Azevedo Giacomina ${ }^{a}$, João Pedro Gonçalves Pacheco $^{a}$, Ricardo Felipe Costa ${ }^{a}$, Christiane Boaventura Lourenço ${ }^{a}$

\section{Resumo}

Objetivo: Identificar as principais causas de atendimento por doenças respiratórias na atenção primária, em Vitória, ES, nos residentes de 0-19 anos, relacionando a gravidade das mesmas com idade e nível socioeconômico, e verificar a variação temporal de frequência desses atendimentos. Métodos: Estudo transversal descritivo dos atendimentos realizados em 2014 nas 30 unidades básicas de saúde do município de Vitória, com dados do sistema de registros eletrônicos Rede Bem Estar. Realizou-se análise descritiva das variáveis sociodemográficas, grupos de diagnóstico e mês de atendimento e regressão logística entre topografia de acometimento do aparelho respiratório e variáveis sociodemográficas. Resultados: Dos 113.252 atendimentos de 0-19 anos, doenças respiratórias constituíram as causas mais frequentes $(28.810,25,43 \%$ do total, $40,18 \%$ dos atendimentos por doença), destacando-se "Infecções agudas das vias aéreas superiores" $(61,35 \%)$, "Doenças crônicas das vias aéreas inferiores" $(14,60 \%)$ e "Outras doenças das vias aéreas superiores" ( $8,69 \%)$. A proporção de atendimentos caiu com a idade e foi maior entre residentes em bairro de menor renda domiciliar média. As vias aéreas superiores foram mais acometidas em todas as faixas etárias, principalmente nas mais avançadas, e nos residentes em bairros de maior renda domiciliar média. Porém, apenas $6 \%$ da variância da distribuição segundo topografia de acometimento do aparelho respiratório foi explicada por faixa etária e nível socioeconômico. Observou-se crescimento dos atendimentos nos meses de março a novembro. Conclusões: Doenças respiratórias são agravos de grande impacto na população pediátrica em Vitória. Recomendam-se medidas de prevenção primária e secundária que levem em consideração a multicausalidade envolvida na determinação dessas doenças.

\begin{abstract}
Objective: To identify the main reasons for visiting a primary health care facility among 0-19 year old residents with respiratory diseases, as well as their frequency, and to associate the severity of those diseases with age and socioeconomic status. Methods: A descriptive cross-sectional study was conducted on all visits to any of the 30 basic health units of the city of Vitória, ES in 2014, using the electronic database Rede Bem Estar. A descriptive analysis was carried out on sociodemographic variables, diagnostic groups and the month of these visits, in addition to logistic regression between the topography of the impairment of the respiratory system and sociodemographic variables. Results: Respiratory diseases were the most frequent reason for visiting the primary health care facility among all 113,252 0-19 years old participants included in the study $(28,810$, corresponding to $25.43 \%$ of the total and $40.18 \%$ of visits due to illness), particularly "acute upper respiratory infections" (61.35\%), "chronic lower respiratory diseases" (14.60\%), and "other diseases of the upper airways" (8.69\%). The months of March to November showed higher frequency of visits, and the visit rates decreased with age, proving to be higher among residents of neighborhoods with lower average income. The upper airways were more affected in all age groups, especially in the older ones, and in residents of higher average income neighborhoods. However, only $6 \%$ of the variance of the distribution based on the topography of the impairment of the respiratory system was explained by age group and socioeconomic status. Conclusions: Respiratory diseases have a major impact on the pediatric population in Vitória. Primary and secondary prevention measures are recommended, taking into account the multi-causality involved in determining these diseases.
\end{abstract}

Como citar: Frauches DO, Lopes IBC, Giacomin HTA, Pacheco JPG, Costa RF, Lourenço $\mathrm{CB}$. Doenças respiratórias em crianças e adolescentes: um perfil dos atendimentos na atenção primária em Vitória/ES. Rev Bras Med Fam Comunidade. 2017;12(39):1-11. http://dx.doi.org/10.5712/rbmfc12(39)1450
Palavras-chave:

Doenças Respiratórias

Perfil de Saúde

Atenção Primária à Saúde

Adolescente

Criança

Keywords:

Respiratory Tract Diseases

Health Profile

Primary Health Care

Adolescent

Child

Fonte de financiamento: declaram não haver.

Parecer CEP:

1.336.228 (EMESCAM), aprovado em 24/11/2015. Conflito de interesses: declaram não haver. Procedência e revisão por pares: revisado por pares. Recebido em: 17/01/2017. Aprovado em: 24/04/2017. 


\section{Resumen}

Objetivo: Identificar las principales causas de enfermedades respiratorias en la atención primaria, en Vitória, ES, para residentes de edades entre 0 y 19 años, relacionar su gravedad con la edad y el estatus socioeconómico y verificar la variación temporal en la frecuencia de estas visitas. Métodos: Estudio descriptivo transversal de las visitas realizadas en el 2014 en las 30 unidades básicas de salud de la ciudad de Vitória, con datos del sistema de registros electrónicos Rede Bem Estar. Se realizó un análisis descriptivo de las variables sociodemográficas, grupos diagnósticos y mes de atención y regresión logística entre la topografía del tracto respiratorio y las variables sociodemográficas. Resultados: De los 113.252 casos de 0 a 19 años de edad, las enfermedades respiratorias fueron las más frecuentes (28.810, $25,43 \%$ del total, $40,18 \%$ de las visitas por enfermedad), especialmente las "Infecciones agudas de las vías respiratorias superiores" (61,35\%), las “Enfermedades crónicas de las vías respiratorias inferiores" (14,60\%) y "Otras enfermedades de las vías aéreas superiores" $(8,69 \%)$. La proporción de asistencias disminuyó con la edad y fue mayor entre los residentes de los vecindarios de ingresos promedios más bajos. Las vías aéreas superiores fueron más afectadas en todos los grupos de edad, especialmente en los más avanzados, y entre los residentes de los barrios de ingreso familiar promedio más alto. Sin embargo, sólo el $6 \%$ de la varianza de la distribución según la topografía del aparato respiratorio se explicó por grupo de edad y estado socioeconómico. Se observó un aumento de las asistencias en los meses de marzo a noviembre. Conclusiones: Las enfermedades respiratorias tienen un gran impacto en la población pediátrica de Vitória. Se recomiendan medidas de prevención primaria y secundaria que tengan en cuenta la multi-causalidad implicada en la determinación de estas enfermedades.

\section{Palabras clave:}

Enfermedades Respiratorias

Perfil de Salud

Atención Primaria de Salud Adolescente

Niño

\section{Introdução}

Doenças respiratórias são um importante problema de saúde pública e representam atualmente a principal causa de mortalidade em crianças menores de cinco anos de idade..$^{1,2}$ De etiologia infecciosa ou não infecciosa, podem acometer tanto as vias aéreas superiores (nariz e fossas nasais, seios perinasais, boca, faringe e laringe), estendendo-se ao ouvido médio, quanto as vias aéreas inferiores (traqueia, brônquios, bronquíolos e pulmões). Esta última localização apresenta, em geral, maior gravidade. ${ }^{3-5}$

Alguns aspectos constituem fatores de risco, como período de inverno, número de moradores no domicílio, baixa condição socioeconômica, idade, tabagismo passivo, baixa escolaridade e pouca idade materna, desnutrição infantil, cultura familiar e maior exposição a poluentes atmosféricos. ${ }^{2}$

As crianças são mais susceptíveis à poluição atmosférica, em comparação com a população adulta, devido a características anatômicas e à imaturidade do sistema fisiológico e imunológico. ${ }^{6}$ Além do controle da poluição, modificações comportamentais como manutenção da amamentação, não exposição da criança à fumaça de cigarro em âmbito doméstico, limpeza contínua da moradia e dos brinquedos com que a criança entra em contato e seguimento correto das orientações médicas, no caso da presença de agravos a saúde, são fundamentais para a prevenção das doenças respiratórias e o melhor manejo dos casos. Também é importante a vacinação, a fim de evitar as doenças infecciosas, ${ }^{7}$ bem como as imunoalérgicas, visto que infecções podem ser fatores desencadeadores para essas doenças. ${ }^{8}$

A atenção primária é a porta de entrada dos usuários no sistema de saúde e está centrada nas unidades básicas de saúde, às quais compete a promoção e a proteção da saúde, na ótica da integralidade, priorizando as demandas e necessidades de saúde de maior prevalência. ${ }^{9}$ Em relação às doenças respiratórias, um dos indicadores da atuação da atenção primária é a proporção de hospitalizações de menores de cinco anos por pneumonia, doença que, ao lado de outras afecções respiratórias, integra a lista brasileira de condições sensíveis à atenção primária. ${ }^{10}$

O elevado impacto das doenças respiratórias na saúde das populações é demonstrado pelo fato de que representam $8 \%$ do total de mortes em países desenvolvidos e $5 \%$ em países em desenvolvimento, 
segundo a Organização Mundial de Saúde, ${ }^{11}$ sendo especificamente as pneumonias responsáveis por 20 a $40 \%$ das hospitalizações dessa população nos países em desenvolvimento. ${ }^{5}$

Sua prevalência é alta em nível nacional, chegando a constituir $16 \%$ das internações no Sistema Único de Saúde, ${ }^{11}$ e acometem expressivamente os menores de 5 anos. ${ }^{2}$ Em Vitória, capital do Espírito Santo, ocupam um lugar expressivo nos atendimentos da rede básica e dos hospitais, ${ }^{12}$ apresentando relação consistente com o perfil misto de poluição atmosférica existente no município, decorrente tanto de fonte veicular como industrial. ${ }^{13}$

Nesse cenário, esse estudo tem como objetivo identificar as principais causas de atendimento por doenças respiratórias na atenção primária, em Vitória, nos residentes de 0-19 anos, relacionando a gravidade das mesmas com idade e nível socioeconômico, e verificar a variação temporal de frequência desses atendimentos.

\section{Métodos}

\section{Local de estudo}

Essa pesquisa foi realizada em Vitória. O município tinha, em 2014, 352.104 habitantes estimados pelo Instituto Brasileiro de Geografia e Estatística (IBGE) ${ }^{14}$ e há vários anos vem apresentando cobertura populacional de $100 \%$ pela atenção primária, de acordo com os Indicadores do Rol de Diretrizes, Objetivos, Metas e Indicadores 2013-2015. ${ }^{15}$ Por outro lado, o número de beneficiários de plano de saúde no município era 234.182 em junho de 2014, segundo dados da Agência Nacional de Saúde Suplementar, ${ }^{16}$ correspondendo a uma cobertura de $66,51 \%$ da população.

Tipo de estudo

Transversal descritivo.

População de estudo

Crianças e adolescentes residentes em Vitória, atendidos em 2014 por médicos e enfermeiros nas 30 unidades básicas de saúde do município, com diagnóstico de doenças respiratórias. Os atendimentos constituíram a unidade de observação. Do total geral de 523.745 atendimentos registrados, foram excluídos 109.648, correspondentes a registros sem CID-10 especificado, sem informação sobre bairro de residência, com residência em bairros de Vitória cuja população é referenciada a outro município ou com residência em outros municípios.

Após esta etapa, foram excluídos 300.845 atendimentos de pessoas de 20 anos e mais e, dos 113.252 atendimentos na faixa etária de 0 a 19 anos, foram excluídos 84.442 por não contemplarem o agravo de interesse, sendo 41.550 deles com CID-10 não indicativo de doença, constante do capítulo "Fatores que influenciam o estado de saúde e o contato com os serviços de saúde" (Z00-Z99).

Ao final do processo de seleção, foram objeto de estudo 27.474 atendimentos de pessoas de 0 a 19 anos, residentes em Vitória, com CID-10 no capítulo doenças do aparelho respiratório (J00-J99), além de 1336 correspondentes a otite média (H65, H66 e H67), totalizando 28.810 atendimentos. 
Fonte e coleta de dados

Os dados foram obtidos do sistema de registros eletrônicos Rede Bem Estar, um complexo de processamento de dados e informações que integra todos os serviços municipais de saúde. ${ }^{17} \mathrm{O}$ sistema contempla desde o prontuário eletrônico do paciente até a emissão de relatórios com algumas variáveis padronizadas, como idade, sexo e bairro de residência da pessoa atendida, unidade e data de atendimento, diagnóstico, procedimento realizado e profissional responsável. Tais relatórios podem conter dados numéricos consolidados na forma de distribuição de frequência simples ou no máximo bivariada, bem como dados detalhados com informação sobre as variáveis disponíveis em relação a cada atendimento, de forma individualizada.

Estes últimos foram utilizados nesta pesquisa, sendo os campos em branco ou com informação deficiente considerados sem informação. Em cada registro, foi inserida no banco informação sobre a renda domiciliar média do bairro de residência, com base no censo demográfico 2010 do IBGE para Vitória. ${ }^{18}$

\section{Análise de dados}

As variáveis de interesse foram idade, renda domiciliar média do bairro de residência e diagnóstico da pessoa atendida, além de mês de ocorrência do atendimento. Idade foi estudada segundo as faixas etárias 0 a 4 anos, 5 a 9 anos, 10 a 14 anos e 15 a 19 anos.

A renda domiciliar média do bairro de residência foi utilizada para estimar nível socioeconômico, informação não disponível na fonte de dados. Esta variável foi categorizada tendo como ponto de corte o valor de 5 salários mínimos, considerando que, segundo o censo demográfico 2010 do IBGE, a população de Vitória residente em bairros com renda domiciliar média até dois salários mínimos era de apenas $0,28 \%$ da população total, enquanto bairros com renda mais de dois a três salários mínimos absorviam somente $9,48 \% .^{18}$

Os diagnósticos, originalmente especificados por categoria da CID-10, foram classificados em sete grupos, a saber: "Doenças do ouvido médio e da mastoide" (H65-H75), "Infecções agudas das vias aéreas superiores" (J00-J06), "Influenza (gripe) e pneumonia" (J09-J18), "Outras infecções agudas das vias aéreas inferiores" (J20-J22), "Outras doenças de vias superiores" (J30-J39), "Doenças crônicas de vias aéreas inferiores" (J40-J47) e "Outras" (J60-J99). Exceto este último, os demais grupos foram, em seguida, dicotomicamente agrupados de acordo com a topografia de acometimento do aparelho respiratório em doenças das vias aéreas superiores (H65-H75, J00-J06 e J30-J39) e doenças das vias aéreas inferiores (J09-J18, J20-J22 e J40-J47). O acometimento de vias aéreas inferiores foi tomado como indicador de gravidade.

Na análise descritiva, foram calculadas frequências absoluta e relativa das variáveis faixa etária, renda domiciliar média do bairro de residência e grupo de diagnósticos. Para quantificar a influência das variáveis sociodemográficas sobre a topografia de acometimento do aparelho respiratório, foi realizada Regressão Logística Binária. A variável dependente foi a topografia de acometimento do aparelho respiratório ( 0 : vias aéreas superiores; 1 : vias aéreas inferiores), e as variáveis independentes foram faixa etária ( 0 a 4 anos, 5 a 9 anos, 10 a 14 anos e 15 a 19 anos) e renda domiciliar média do bairro de residência (0: até 5 salários mínimos; 1: mais de cinco salários mínimos). 
As variáveis foram selecionadas por eliminação para trás pelo teste de Wald. O teste de Qui quadrado omnibus $(p<0,05)$ e o teste de Hosmer e Lemeshow $(p>0,05)$ foram usados para determinar o ajuste do modelo, enquanto que o $\mathrm{R}^{2}$ de Nagelkerke foi usado com índice de variância explicada, em que quanto maior é o valor de $\mathrm{R}^{2}$ melhor é o modelo. A codificação das variáveis e a análise estatística foram conduzidas com Statistical Package for the Social Sciences (SPSS), versão 23.0.

\section{Aspectos éticos}

O projeto deste estudo foi aprovado pelo Comitê de Ética em Pesquisa da Escola Superior de Ciências da Santa Casa de Misericórdia de Vitória (CAAE 44858215.6.0000.5065).

\section{Resultados}

Excluídas causas de atendimento não indicativas de doença, as doenças respiratórias constituíram o motivo mais frequente nos atendimentos da atenção primária à população de 0 a 19 anos residente em Vitória, perfazendo $28.810(25,43 \%)$ do total de 113.252 atendimentos a pessoas da faixa etária de interesse. Dos 71.702 atendimentos por doença, nessa faixa, representaram 40,18\%.

Conforme apresentado na Tabela 1, os atendimentos concentraram-se na faixa etária de 0 a 4 anos $(60,07 \%)$ e nos residentes em bairros com menor renda domiciliar média $(72,45 \%)$. Entre os grupos de diagnóstico, o mais frequente foi o das "Infecções agudas das vias aéreas superiores" (61,35\%), seguido de "Doenças crônicas das vias aéreas inferiores" (14,60\%) e de "Outras doenças das vias aéreas superiores" $(8,69 \%)$.

Tabela 1. Atendimentos de crianças e adolescentes com doenças respiratórias na atenção primária, por faixa etária e por nível de renda domiciliar média do bairro de residência, segundo grupo de diagnósticos - Vitória, ES - 2014.

\begin{tabular}{|c|c|c|c|c|c|c|c|c|}
\hline $\begin{array}{l}\text { Grupos de } \\
\text { diagnóstico } \\
\text { (CID-10) }\end{array}$ & $\begin{array}{l}\text { Doenças } \\
\text { do ouvido } \\
\text { médio e da } \\
\text { mastoide }\end{array}$ & $\begin{array}{c}\text { Infecções } \\
\text { agudas das } \\
\text { vias aéreas } \\
\text { superiores }\end{array}$ & $\begin{array}{c}\text { Influenza } \\
\text { [gripe] e } \\
\text { pneumonia }\end{array}$ & $\begin{array}{c}\text { Outras } \\
\text { infecções } \\
\text { agudas das } \\
\text { vias aéreas } \\
\text { inferiores }\end{array}$ & $\begin{array}{c}\text { Outras } \\
\text { doenças das } \\
\text { vias aéreas } \\
\text { superiores }\end{array}$ & $\begin{array}{c}\text { Doenças } \\
\text { crônicas das } \\
\text { vias aéreas } \\
\text { inferiores }\end{array}$ & Outras & Total \\
\hline \multicolumn{9}{|l|}{ Faixa etária } \\
\hline 0 a 4 & $\begin{array}{c}815 \\
(4,71 \%)\end{array}$ & $\begin{array}{c}10163 \\
(58,72 \%)\end{array}$ & $\begin{array}{c}1028 \\
(5,94 \%)\end{array}$ & $\begin{array}{c}1527 \\
(8,82 \%)\end{array}$ & $\begin{array}{c}926 \\
(5,35 \%)\end{array}$ & $\begin{array}{c}2840 \\
(16,41 \%)\end{array}$ & $\begin{array}{c}8 \\
(0,05 \%)\end{array}$ & 17307 \\
\hline 5 a 9 & $\begin{array}{c}281 \\
(5,05 \%)\end{array}$ & $\begin{array}{c}3467 \\
(62,34 \%)\end{array}$ & $\begin{array}{c}176 \\
(3,16 \%)\end{array}$ & $\begin{array}{c}101 \\
(1,82 \%)\end{array}$ & $\begin{array}{c}678 \\
(12,19 \%)\end{array}$ & $\begin{array}{c}856 \\
(15,39 \%)\end{array}$ & $\begin{array}{c}2 \\
(0,04 \%)\end{array}$ & 5561 \\
\hline 10 a 14 & $\begin{array}{c}138 \\
(4,14 \%)\end{array}$ & $\begin{array}{c}2113 \\
(63,38 \%)\end{array}$ & $\begin{array}{c}82 \\
(2,46 \%)\end{array}$ & $\begin{array}{c}64 \\
(1,92 \%)\end{array}$ & $\begin{array}{c}561 \\
(16,83 \%)\end{array}$ & $\begin{array}{c}372 \\
(11,16 \%)\end{array}$ & $\begin{array}{c}4 \\
(0,12 \%)\end{array}$ & 3334 \\
\hline 15 a 19 & $\begin{array}{c}102 \\
(3,91 \%)\end{array}$ & $\begin{array}{c}1932 \\
(74,08 \%)\end{array}$ & $\begin{array}{c}55 \\
(2,11 \%)\end{array}$ & $\begin{array}{c}36 \\
(1,38 \%)\end{array}$ & $\begin{array}{c}339 \\
(13,00 \%)\end{array}$ & $\begin{array}{c}137 \\
(5,25 \%)\end{array}$ & $\begin{array}{c}7 \\
(0,27 \%)\end{array}$ & 2608 \\
\hline Mais de 5 & $\begin{array}{c}350 \\
(4,41 \%)\end{array}$ & $\begin{array}{c}4969 \\
(62,61 \%)\end{array}$ & $\begin{array}{c}265 \\
(3,34 \%)\end{array}$ & $\begin{array}{c}233 \\
(2,94 \%)\end{array}$ & $\begin{array}{c}989 \\
(12,46 \%)\end{array}$ & $\begin{array}{c}1124 \\
(14,16 \%)\end{array}$ & $\begin{array}{c}6 \\
(0,08 \%)\end{array}$ & 7936 \\
\hline Total & $\begin{array}{c}1336 \\
(4,64 \%)\end{array}$ & $\begin{array}{c}17675 \\
(61,35 \%)\end{array}$ & $\begin{array}{c}1341 \\
(4,65 \%)\end{array}$ & $\begin{array}{c}1728 \\
(6,00 \%)\end{array}$ & $\begin{array}{c}2504 \\
(8,69 \%)\end{array}$ & $\begin{array}{c}4205 \\
(14,60 \%)\end{array}$ & $\begin{array}{c}21 \\
(0,07 \%)\end{array}$ & 28810 \\
\hline
\end{tabular}

${ }^{*}$ Renda domiciliar média do bairro de residência, em salários mínimos. 
A proporção de atendimentos pelos diversos grupos de diagnóstico variou conforme a faixa etária (Tabela 1). Em todas, predominaram as "Infecções agudas das vias aéreas superiores", cuja proporção cresceu com a idade. Nota-se que "Doenças do ouvido médio e da mastoide" e "Outras doenças das vias aéreas superiores" seguiram padrão de crescimento semelhante, as primeiras até os 9 anos e as últimas até os 14 anos. Já o inverso ocorreu com "Influenza e pneumonia", bem como com "Outras infecções agudas das vias aéreas inferiores" e com as "Doenças crônicas das vias aéreas inferiores", nas quais houve decréscimo de proporção com o aumento da idade.

Em relação à renda domiciliar média do bairro de residência (Tabela 1), os residentes em bairros de maior nível de renda apresentaram frequência de "Infecções agudas das vias aéreas superiores" um pouco maior e de "Outras doenças das vias aéreas superiores" bem maior do que os residentes em bairros de menor nível de renda. Os demais grupos de diagnósticos predominaram nos residentes em bairros de menor nível de renda.

A maioria dos grupos de diagnóstico apresentou tendência de crescimento a partir dos primeiros meses do ano, com pico de ocorrência em abril, e novo crescimento a partir de maio até o início do segundo semestre, com pico em julho ou agosto (Figura 1). O grupo das "Outras doenças das vias aéreas superiores" alcançou o primeiro pico somente em junho e o segundo, em setembro, enquanto o grupo "Outras" não apresentou padrão regular. Observa-se que as "Infecções agudas das vias aéreas superiores" atingiram o ápice de ocorrência no segundo semestre, ao contrário dos outros grupos.

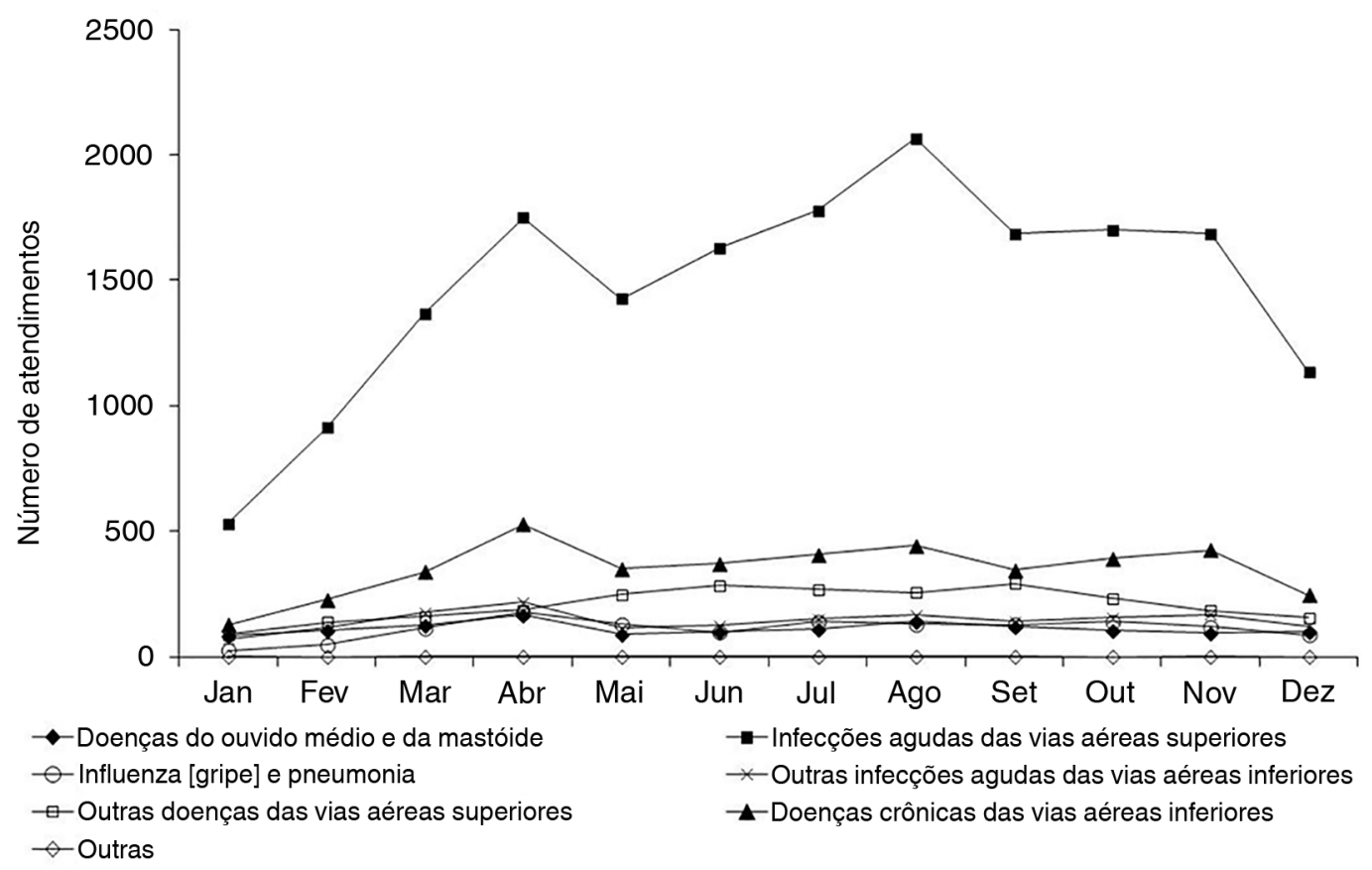

Figura 1. Frequência mensal dos atendimentos de crianças e adolescentes com doenças respiratórias na atenção primária, segundo grupo de diagnósticos - Vitória, ES - 2014.

Como pode ser observado na Tabela 2, a proporção de acometimento das vias aéreas inferiores, indicador de gravidade, caiu com o aumento da idade e do nível de renda. 
Tabela 2. Frequência e percentagem da topografia de acometimento das vias aéreas de acordo com a faixa etária e nível socioeconômico das crianças e adolescentes atendidos na atenção primária em Vitória, ES - 2014.

\begin{tabular}{|c|c|c|c|c|}
\hline \multirow{3}{*}{ Variáveis } & \multicolumn{4}{|c|}{ Doenças respiratórias* } \\
\hline & \multicolumn{2}{|c|}{ Vias aéreas superiores } & \multicolumn{2}{|c|}{ Vias aéreas inferiores } \\
\hline & No & $\%$ & No & $\%$ \\
\hline \multicolumn{5}{|c|}{ Faixa etária } \\
\hline 0 a 4 & 11904 & 68,81 & 5395 & 31,19 \\
\hline 5 a 9 & 4426 & 79,62 & 1133 & 20,38 \\
\hline 10 a 14 & 2812 & 84,44 & 518 & 15,56 \\
\hline 15 a 19 & 2373 & 91,23 & 228 & 8,77 \\
\hline \multicolumn{5}{|c|}{ Renda domiciliar média** } \\
\hline Até 5 & 15207 & 72,90 & 5652 & 27,10 \\
\hline Mais de 5 & 6308 & 79,55 & 1622 & 20,45 \\
\hline
\end{tabular}

*Excluídas "Outras"; ** Renda domiciliar média do bairro de residência, em salários mínimos.

Este fato foi confirmado pela análise de regressão logística (Tabela 3), que demonstrou um maior fator de proteção para faixa etária entre 15 a 19 anos (para aproximadamente 24 casos na faixa etária de referência, há somente um adolescência tardia) e que residir em bairro com renda domiciliar média maior que 5 salários mínimos diminui a chance de acometimento das vias aéreas inferiores (cerca de um caso para mais de dois do nível de renda mais baixo). No entanto, faixa etária e nível socioeconômico influenciaram apenas $6 \%$ da variância total das doenças respiratórias categorizadas segundo a topografia de acometimento do aparelho respiratório.

Tabela 3. Influência da faixa etária e nível socioeconômico na chance (OR) de acometer infecções nas vias aéreas inferiores em crianças e adolescentes atendidas pela atenção primária em Vitória, ES - 2014.

\begin{tabular}{ll}
\hline Variáveis & Razão de odds (IC 95\%) \\
\hline Faixa etária & \\
\hline 0 a 4 & Referência \\
5 a 9 & $0,57(0,53-0,61)$ \\
10 a 14 & $0,41(0,37-0,45)$ \\
15 a 19 & $0,22(0,19-0,25)$ \\
\hline
\end{tabular}

Renda domiciliar média*

Até 5

Referência

Mais de 5

$0,71(0,67-0,76)$

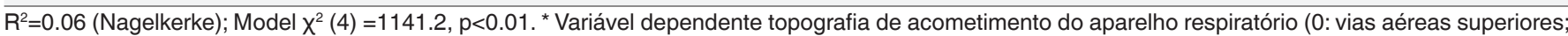
1 : vias aéreas inferiores). ${ }^{*}$ Renda domiciliar média do bairro de residência, em salários mínimos.

\section{Discussão}

Observou-se, no presente estudo, que as doenças respiratórias foram a principal causa de atendimento na atenção primária em Vitória, na população de 0 a 19 anos, em 2014, o que confirma o grande impacto deste agravo na população pediátrica do município e está em consonância com a alta prevalência dessas doenças em âmbito nacional. ${ }^{11}$ 
A grande proporção de atendimentos por doenças respiratórias encontrada em Vitória pode estar relacionada à qualidade do ar no município. Na capital do Espírito Santo há uma exposição mista aos poluentes atmosféricos, com contribuição tanto de dejetos industriais como veiculares, tendo sido observada relação entre doenças respiratórias e particulado PM10, que representa o poluente de maior associação com internações por causas respiratórias. ${ }^{13}$

Foi observada maior frequência de atendimentos na faixa etária de zero a quatro anos e em residentes em bairros de menor renda domiciliar média, com crescimento da frequência a partir dos primeiros meses do ano.

A maior vulnerabilidade biológica das crianças de zero a quatro anos explica o alto percentual de atendimentos encontrado nessa faixa etária, em consonância com a literatura. Em crianças, o epitélio das vias aéreas é mais permeável aos poluentes e as defesas pulmonares contra essas partículas não estão completamente desenvolvidas. Além disso, têm, proporcionalmente, um maior volume de ar circulante em suas vias aéreas, pois possuem uma taxa ventilatória maior em função do metabolismo mais acelerado, da maior exposição ao ambiente externo e da prática mais exuberante de atividade física. ${ }^{6}$

Adicionalmente, crianças mais jovens possuem menor número de alvéolos, canais colaterais de ventilação menos desenvolvidos (ou ausentes, dependendo da idade), vias aéreas menores e mais estreitas, laringe localizada mais superiormente, menos cartilagem para promover a sustentação das vias aéreas, entre outros fatores, o que facilita a instalação de microorganismos em suas vias aéreas. ${ }^{19}$ Têm, também, o sistema imunológico ainda imaturo, o que leva a uma maior frequência de doenças potencialmente mais graves ou de complicações de infecções de vias aéreas superiores. ${ }^{20}$

Quanto ao nível socioeconômico, os resultados também estão de acordo com a literatura, visto que os principais fatores de risco para doenças respiratórias, especialmente as mais graves, são a baixa escolaridade materna, aglomeração familiar, condições precárias de moradia e tabagismo materno. ${ }^{4}$

Além da maior vulnerabilidade dos residentes em bairros de menor renda domiciliar média, o predomínio dos atendimentos neste grupo populacional se justifica também pela existência, para os de maior nível socioeconômico, de outras opções de acesso a serviços de saúde, como planos de saúde, convênios empresariais ou de outros tipos, determinando, para esse último grupo, subregistro de doenças de uma maneira geral nas unidades básicas de saúde. ${ }^{16,21}$

Dentre as doenças respiratórias, a proporção de atendimentos por "Infecções agudas das vias aéreas superiores" variou com a faixa etária, mas em todas mostrou-se muito superior aos demais grupos de diagnóstico, como reflexo de sua alta prevalência. As infecções respiratórias agudas possuem uma representatividade mundial de 20 a $40 \%$ das consultas em serviços de pediatria e 12 a $35 \%$ das internações hospitalares, o que reitera a importância do bom manejo clínico dessas patologias. ${ }^{5,22,23}$

Em seguida, vieram as "Doenças crônicas das vias aéreas inferiores", entre as quais encontram-se diagnósticos como bronquite e asma, doenças imunoalérgicas também relativamente comuns em crianças e adolescentes. ${ }^{19,24} \mathrm{~A}$ asma é uma doença de difícil controle clínico e, no Brasil, sua prevalência estimada em crianças é de $20 \%$. Outros fatores importantes, além da má aderência ao tratamento, são a dificuldade de acesso ao sistema básico de saúde e o baixo nível socioeconômico. ${ }^{25}$ Nota-se que as faixas etárias mais jovens, mais vulneráveis aos quadros graves, foram mais acometidas por doenças de vias aéreas inferiores. 
Embora os atendimentos por "Infecções agudas das vias aéreas superiores" tenham predominado em ambos os níveis de renda estudados, o nível de renda mais alto apresentou proporção um pouco maior este grupo de diagnóstico. Tal diferença, bem maior, também se deu quanto a "Outras doenças das vias aéreas superiores", enquanto o menor nível de renda mostrou-se mais afetado pelos demais grupos, principalmente "Influenza [gripe] e pneumonia" e "Outras infecções agudas das vias aéreas inferiores", um indicativo de sua maior vulnerabilidade.

Porém, mesmo demonstrada a existência de associação entre faixa etária e nível socioeconômico e a topografia de acometimento do aparelho respiratório (vias aéreas superiores e vias aéreas inferiores), a variância encontrada em nosso estudo, de $6 \%$, indica que essas duas variáveis apresentam pouca influência como fator de risco global para a gravidade das doenças respiratórias, sendo a multicausalidade uma questão importante. Dessa forma, é necessário considerar os demais fatores de risco, como os comportamentais, ambientais e genéticos, no manejo das doenças respiratórias.

Os atendimentos por doenças respiratórias apresentaram polarização entre os meses de março a novembro, mais uma vez coincidindo com a literatura. Tal padrão, como um todo, pode ser explicado tanto pelo fato de esse ser o período de atividades escolares, aumentando o risco de transmissão das doenças devido ao maior número de contactantes, como também pela maior permanência em ambientes fechados e com aglomeração de pessoas, condições geralmente associadas às menores temperaturas. ${ }^{19,22,24}$

A relação entre baixas temperaturas, aglomerações e doenças respiratórias tem substancial ancoragem no fato de que os atendimentos por "Infecções de vias aéreas superiores" apresentaram importante aumento de frequência no período referente ao inverno, com pico em agosto.

Mesmo com grande representatividade populacional, limitações importantes desse estudo consistem na utilização de dados secundários, sujeitos a falhas de registro, e em sua abrangência restrita aos usuários da rede de atenção primária do município, no qual é alta a cobertura de planos de saúde privados.

Adicionalmente, nesse estudo não é possível avaliar a interferência do grau de resolutividade ambulatorial de cada unidade básica, fator que pode alterar o número de atendimentos em relação a um problema de saúde e que talvez afete de forma diferente as diversas unidades. Desse modo, a territorialização existente na atenção primária à saúde pode representar um viés.

Finalmente, a utilização da variável renda domiciliar média do bairro de residência para estimar o nível socioeconômico produz falácia ecológica, por se tratar de um dado agregado, podendo a heteregeneidade de renda dentro de um mesmo bairro afetar a demanda aos serviços públicos de saúde. Além disso, o nível de renda da população do município (menos de 10\% residente em bairros com renda média domiciliar de até três salários mínimos) condicionou a utilização de um ponto de corte relativamente alto (cinco salários mínimos), talvez subestimando a associação entre nível de renda e acometimento de vias aéreas inferiores.

\section{Conclusão}

Este estudo confirma, em Vitória, as doenças respiratórias como agravos de grande impacto na saúde da população pediátrica, principalmente a mais jovem. A distribuição dos atendimentos por idade ocorreu conforme descrito na literatura, o mesmo acontecendo em relação a nível socioeconômico, aos grupos de diagnóstico e à distribuição temporal. 
Embora menor faixa etária e menor nível socioeconômico tenham se mostrado fatores de risco para doenças das vias aéreas inferiores, tiveram pequeno papel na explicação do acometimento respiratório baixo, sendo necessário estudar a magnitude de outros determinantes.

Considerando a epidemiologia local, recomendam-se medidas de prevenção primária e secundária em relação às doenças respiratórias, tais como controle da poluição atmosférica, ventilação adequada dos ambientes coletivos, educação em saúde, vacinação contra influenza e implementação de protocolos de tratamento para infecções respiratórias agudas e para asma nas unidades básicas de saúde.

\section{Referências}

1. Fioravanti C. Progress and challenges for respiratory health in Brazil. Lancet Respir Med. 2015;3(5):348-9. DOI: http://dx.doi.org/10.1016/S2213-2600(15)00152-6

2. Prato MIC, Silveira A, Neves ET, Buboltz FL. Doenças respiratórias na infância: uma revisão integrativa. Rev Soc Bras Enferm Ped. 2014;14(1):33-9.

3. Sonego M, Pellegrin MC, Becker G, Lazzerini M. Risk factors for mortality from acute lower respiratory infections (ALRI) in children under five years of age in low and middle-income countries: a systematic review and meta-analysis of observational studies. PLoS One. 2015;10(1):e0116380. DOI: http://dx.doi.org/10.1371/journal.pone.0116380

4. Prietsch SOM, Fischer GB, Cesar JA, Fabris AR, Mehanna H, Ferreira THP, et al. Doença aguda das vias aéreas inferiores em menores de cinco anos: influência do ambiente doméstico e do tabagismo materno. J Pediatr (Rio J.). 2002;78(5):415-22. DOI: http://dx.doi.org/10.1590/S0021-75572002000500013

5. Duarte DMG, Botelho C. Perfil clínico de crianças menores de cinco anos com infecção respiratória aguda. J Pediatr (Rio J.). 2000;76(3):207-12. DOI: http://dx.doi.org/10.2223/JPED.55

6. Salvi S. Health effects of ambient air pollution in children. Paediatr Respir Rev. 2007;8(4):275-80. DOI: http://dx.doi. org/10.1016/j.prrv.2007.08.008

7. Neves RG, Böhm AW, Costa CS, Flores TR, Soares ALG, Wehrmeister FC. Cobertura da vacina meningocócica C nos estados e regiões do Brasil em 2012. Rev Bras Med Fam Comunidade. 2016;11(38):1-10. DOI: http://dx.doi.org/10.5712/ rbmfc11(38)1122

8. Sociedade Brasileira de Pneumologia. Diretrizes da Sociedade Brasileira de Pneumologia e Tisiologia para o manejo da asma - 2012. J Bras Pneumol. 2012;38(Suppl 1):S1-46.

9. Brasil. Ministério da Saúde. PNAB - Política Nacional de Atenção Básica. Brasília: Ministério da Saúde;2012. [acesso 2017 Maio 3]. Disponível em: http://189.28.128.100/dab/docs/publicacoes/geral/pnab.pdf

10. Alfradique ME, Bonolo PF, Dourado I, Lima-Costa MF, Macinko J, Mendonça CS, et al. Internações por condições sensíveis à atenção primária: a construção da lista brasileira como ferramenta para medir o desempenho do sistema de saúde (Projeto ICSAP - Brasil). Cad Saúde Pública. 2009;25(6):1337-49. DOI: http://dx.doi.org/10.1590/S0102-311X2009000600016

11. Bernat AC, Oliveira MC, Rocha GC, Boing AF, Peres KG. Prevalência de sintomas respiratórios e fatores associados: estudo de base populacional em adultos de Lages, Santa Catarina, Brasil. Cad Saúde Pública. 2009;25(9):1907-16. DOI: http://dx.doi.org/10.1590/S0102-311X2009000900005

12. Castro HA, Hacon S, Argento R, Junger WL, Mello CF, Castiglioni Júnior N, et al. Air pollution and respiratory diseases in the Municipality of Vitória, Espírito Santo State, Brazil. Cad. Saúde Pública. 2007;23(Suppl.4):630-42. DOI: http://dx.doi.org/10.1590/S0102-311X2007001600023

13. Freitas CU, Ponce de Leon A, Juger W, Gouveia N. Air pollution and its impacts on health in Vitoria, Espirito Santo, Brazil. Rev Saúde Pública. 2016;50:4. DOI: http://dx.doi.org/10.1590/S1518-8787.2016050005909

14. Brasil. Instituto Brasileiro de Geografia e Estatística (IBGE) [homepage na internet]. Estimativas populacionais para os municípios brasileiros em 01.07.2014. [acesso 2016 Out 29]. Disponível em: http://www.ibge.gov.br/home/estatistica/ populacao/estimativa2014/estimativa_dou.shtm 
15. Brasil. Ministério da Saúde. Departamento de Informações e Informática do SUS. Rol de diretrizes, objetivos, metas e indicadores 2013-2015: edição 2015. [acesso 2016 out 29]. Disponível em: http://tabnet.datasus.gov.br/cgi/deftohtm. exe?pacto/2015/cnv/coapcires.def.

16. Brasil. Agência Nacional de Saúde Suplementar [homepage na internet]. Assistência Médica por Competência segundo Faixa etária [acesso 2016 Dez 7]. Disponível em: http://www.ans.gov.br/anstabnet/cgi-bin/tabnet?dados/tabnet_02.def

17. Prefeitura Municipal de Vitória. Lei 8601, de 23 de dezembro de 2013. Dispõe sobre o sistema rede bem estar, a certificação dos registros, e dá outras providências. Vitória: Diário Oficial, 2 jan. 2014. [acesso 2016 Out 29]. Disponível em: http:// leismunicipais.com.br/a/es/v/vitoria/lei-ordinaria/2013/861/8601/lei-ordinaria-n-8601-2013-dispoe-sobre-o-sistema-redebem-estar-a-certificacao-dos-registros-e-da-outras-providencias?q=Lei\%208601

18. Secretaria de Gestão Estratégica. Vitória bairro a bairro. Vitória: Prefeitura Municipal de Vitória; jan 2013. [acesso 2016 Dez 7]. Disponível em: http://legado.vitoria.es.gov.br/regionais/geral/publicacoes/Vitoria_bairro_bairro/Vit\%C3\%B3ria_ bairro_\%20a_bairro.pdf

19. Kliegman R, Jenson HB, Behrman RE. Nelson - Tratado de Pediatria. 19a ed. Rio de Janeiro: Elsevier; 2013.

20. Pereira BB, Limongi JE. Epidemiologia de desfechos na saúde humana relacionados à poluição atmosférica no Brasil: uma revisão sistemática. Cad Saúde Colet. 2015;23(2):91-100. DOI: http://dx.doi.org/10.1590/1414-462X201400050103

21. Turrini RNT, Lebrão ML, Cesar CLG. Resolutividade dos serviços de saúde por inquérito domiciliar: percepção do usuário. Cad Saúde Pública. 2008;24(3):663-74. DOI: http://dx.doi.org/10.1590/S0102-311X2008000300020

22. Pitrez PMC, Pitrez JLB. Infecções agudas das vias aéreas superiores: diagnóstico e tratamento ambulatorial. J Pediatr. 2003;70(Suppl.1):S77-86. DOI: http://dx.doi.org/10.1590/S0021-75572003000700009

23. Mouro A, Hidal LBT, Martino MDV, Pasternark J. Prevalência das infecções de vias aéreas superiores em um hospital terciário na cidade de São Paulo. Einstein (São Paulo). 2010;8(2):197-9. DOl: http://dx.doi.org/10.1590/s1679-45082010ao1348

24. Miranda DC. Prevalência de asma e sintomas respiratórios no município de Vitória (ES): comparação entre duas áreas com diferentes fontes de poluição atmosférica identificadas através do biomonitoramento [Tese de doutorado]. São Paulo: Faculdade de Medicina da Universidade de São Paulo;2008. DOI:http://dx.doi.org/10.11606/T.5.2008.tde-30102008-161613

25. Urrutia-Pereira M, Avila J, Solé D. The Program for the Prevention of Childhood Asthma: a specialized care program for children with wheezing or asthma in Brazil. J Bras Pneumol. 2016;42(1):42-7. DOI: http://dx.doi.org/10.1590/S180637562016000004480

a Escola Superior de Ciências da Saúde Santa Casa de Misericórdia de Vitória (EMESCAM). Vitória, ES, Brasil. dianafrauches@emescam.br (Autora correspondente); isabelabittencoutinho@gmail.com; henrique.ton@ hotmail.com; jpgp123@hotmail.com; ricardo_felipe_costa@hotmail.com; christiane.lourenco@emescam.br 